\section{RECENT ADVANCES IN UTILIZATION OF THE R-MATRIX PARAMETERS FOR REACTOR APPLICATIONS*}

ANL/CP -71459

DE91 013406

R. N. Hwang

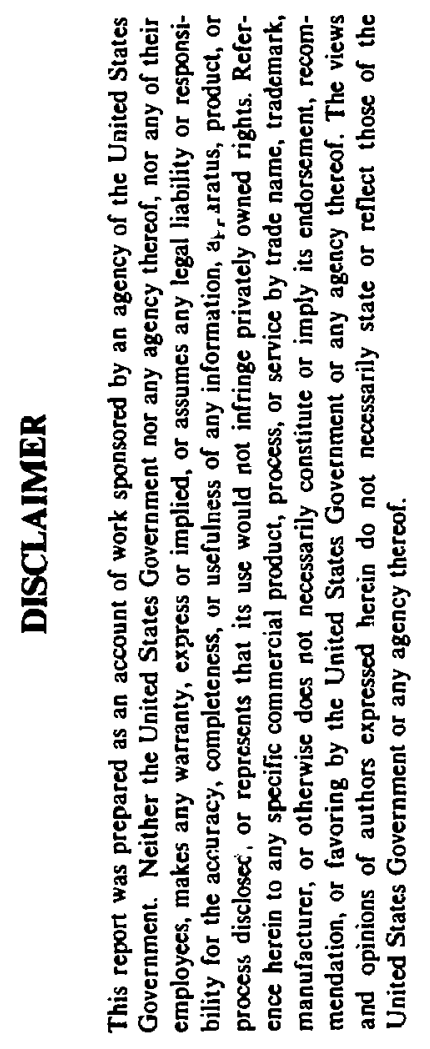

\author{
Argonne National Laboratory \\ 9700 S. Cass Ave \\ Argonne, IL 60439, USA
}

\begin{abstract}
The submitted manuxcript has been euthored by cinntractor of the U.S. Government under contract No. W-31-109ENG-38. Acrordingly, the U.S. Government retsins a Accordingly, the U. S. Government reasins a nonexclusive, royslty-free lieents to publich or reproduce the published lorm of this
contribution, of allow others to do $\infty$, for
\end{abstract} U. S. Government purposes.

"Work performed under the auspices of the U.S. Department of Energy, Nuclear Energy Program under the Contract W-31-109-ENG-38. 
RECENT ADVANCES IN UTILIZATION OF THE R-MATRIX PARAMETERS FOR REACTOR APPLICATIONS*

\author{
R. N. Hwang
}

Argonne National Laboratory

9700 S. Cass Ave

Argonne, IL 60439, USA

\begin{abstract}
Abs iract: A simplified version of the rigorous pole representation of cross sections has been developed to facilitate utilization of the newly released ENDF/B VI resonance data based on the Reich-Mooze parameters for reactor applications. The procedure is equivalent to the extraction of the Humblet-Rosenfeld-type parameters and the associated 'background' term explicitly from the rigorous pule and residue parameters which, in turn, are converted from a given set of the Reich-Koore parameters. The computational efficiency and its amenability to the existing reactor codes are significanily enhanced by the introduction of the percinent analytic continuation in place of the smooth 'background' term via the non-linear least square fitting. The method has been successfully applied to all major nuclides examined and the results are presented.
\end{abstract}

\section{Introduction}

Two special features of the rewiy released ENDE/B VI resonance data that will have profound impacts on the cross section processing codes for reactor applications are the dramatic expansion of the resolved energy regions and the availability of the R-Matrix parameters based on the Reich. Moore formalism ${ }^{2}$ for most major nuclides of practical interest. While the availability of the new resonance data has all but eliminated the longstanding difficulties attributed to the lack of sufficient resolved resonance information and the inadequacy of the Breit-Wigner single level representation for the closely spaced resonances, one obvious question of great practical concern is the compatibility of the new resonance representation with the existing reactor methodologies based almost exclusively on the traditional formalisms.

To facilitate the efficient utilization of the new R-matrix data, a simplified version of the rigorous pole representation described previous $1 \mathrm{y}^{3}$ has been developed so that the rigor of the RefchMoore cross sections and the tradicional feature of Doppler broadening via the Voigt profile essential to many existing codes can be preserved simultaneously. Extensive calculations have been carried out to dewonstrate the viability of the proposed method and the results will be presented.

\section{Natural extension of Rigorous} Pole Representation

The theoretical justification of the rigorous pole representation is based on the rationale that the collision matrix must be single-valued and meromorphic in the momentum space. Any function that exhibits such properties must be a rational function according to the well known theorem in complex analysis. Thus, one obtains via partial fraction ${ }^{3}$.

$$
\sigma_{x}=\frac{1}{E} \sum_{i, J} \sum_{i=1}^{N} \sum_{j=1}^{2(1+1)} \Re e\left\{R_{i, J, j, \lambda}^{(x)} \cdot \frac{(-i)}{P_{\lambda}^{(j)}-\sqrt{E}}\right\}
$$

where $\mathbf{x}$ denotes the type of reaction under cor:sideration and $N$ is the total number of $k=0 \mathrm{~mm}$ $R$-matrix resonances. The geruine energy inciependent pole and residue paranecers can be obzained from any given set of the Reich-Moore parameters using the WHOPPER code. ${ }^{3}$ The expression leads imrediately to the well-known Voigt profiles winen subject to Doppler-broadening. However, the presence of $2(\ell+1)$ terms for each resonance to be evaluated in momentum domain is obviously unde. sirable from the point-of-view of compuing efficiency, storage requirement and its amencioility to existing cocies. In the following discussion, it will be shown how the problem can be alleviated.

The $2(\ell+1) N$ poles for a given $\ell$ and $J$ defined in Eq. 1 can be divided into two distinct classes. There are $2 \mathrm{~N}$ s-wave-like poles with distinct spacings while the remaining $22 \mathbb{N}$ poles are closely spaced independent of how well separated the input Reich-Moore rasonances are. Such characteristics can be best visualized by examin. ing the denominator of the rational function leading to Eq. 1 .

$$
P_{2(l+1) N}(\sqrt{E})=P_{2 N}^{(1)}(\sqrt{E}) \cdot P_{2 E N}^{(2)}(\sqrt{E})
$$

where $R_{2 N}^{(1)}(\sqrt{E})$ is a polynomial with its compiex coefficients strongly dependent on the inpue resonance parameters whereas the coefficients of $\mathrm{p}_{2 \mathrm{~N}}^{(2)}(\sqrt{E})$ are dominated by terms involvirg $k_{2} a$ with a - channel radius and $k_{s}=2.197 \times 10^{-3}$ $(A / 1+A)]$.

Thus, $P_{2 \mathrm{NN}}^{(2)}(\sqrt{E})$ reflects the higher orcier energy dependence of the penetration and sifif factors and tts roots are barely distinguisiajle. In addition, the magnicude of their imasiary components becomes of the order of $1 / k_{a} a i s$ the limit of small neutron widths. Such charac $= \pm z^{*}$. tics had already been illustrated analytically for the cases of a single level in Ref. 3. The sid of these pole terms involving poles with exceecingly large 'width' (or imaginary part) can be considered as the background contribution to the cross section with weak $\sqrt{E}$ deperdence. 
In contrast, the roots of $\mathrm{P}_{2 \mathrm{X}}^{(1)}(\sqrt{E})$ are distinct and always appear in pairs with their real components opposite in signs but not necessarily equal in magnitude spanning over

$\left(\sqrt{E_{\min }} \leq \sqrt{E} \leq \sqrt{E_{\max }}\right.$ and $\left.-\sqrt{E_{\max }} \leq \sqrt{E} \leq-\sqrt{E_{\min }}\right)$ on the real axis of the momentum domain. Let

$\mathrm{p}_{\lambda}^{(1)}$ and $\mathrm{p}_{\lambda}^{(2)}$ be poles with positive and negative real components, respectively. It is important to note that the actual interval of interest in computing cross sections is half of the interval taken to be $\left(\sqrt{E_{l}} \leq \sqrt{E} \leq \sqrt{E_{u}}\right)$ where $E_{u}\left(<E_{\max }\right)$ and $E_{l}\left(>E_{\text {min }}\right.$ ) are the upper and lower resolved region boundaries, respectively. Because of their short range nature of fluctuation, all terms involving $p_{\lambda}^{(2)}$ conscitute another smooth component to $o_{x}$ that reflects the contributions from the tails of rutying poles outside the interval of practical interest.

Let $q_{l}^{(x)}(\sqrt{E})$ denote the contributions from those $2 E \mathrm{~N}$ terms involving poles with giant 'width'. By taking advantage of the characteris. tics of poles described above, Eq. 1 can be cast inco the same form as that of tumblet-Rosenfeld."

$$
\begin{aligned}
& \sigma_{x}=\frac{1}{E} \sum_{i} \operatorname{Re}\left\{\sum_{F} \sum_{\lambda=1}^{N}\left[R_{l, J, 1,1}^{(x)} \cdot \frac{2(-i) \sqrt{E}}{\left(p_{\lambda}^{(1)}\right)^{2}-E}\right]\right. \\
& \left.+s_{i}^{(x)}(\sqrt{E})+g_{i}^{(x)}(\sqrt{E}) \cdot s_{i}\right\}
\end{aligned}
$$

where

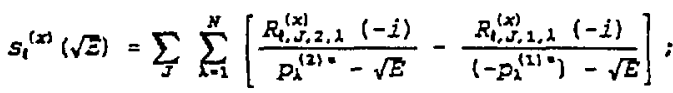

$$
\begin{aligned}
& \delta_{0}=0, \delta_{1}=1 \text { for } \ell>0
\end{aligned}
$$

Hence, for a given range of practical interest, the rigorous pole representation can be viewed as a combination of a 'fluctuating' term consisting of $N$ poles with He $p_{\lambda}^{(1)}>0$ expressed in the energy domain consistent with the tradi. tional formalisms and two 'non-fluctuating' terms actribuced to the tails of outlying poles with negative real component and the poles with extremely large 'width' (or $\left|\Im m p_{\lambda}^{(j)}\right|$ ) for $\ell>0$ states respectively. The striking behavior of the 'fluctuating' and 'non-fluctuating' components have been confirmed in recent calculations for all rajor nuclides specified by the Reich-Moore parameters in the ENDF/B VI files.

Eq. 3 represents no more than an empty form unless the background terms can be evaluated efficiently. The smooth behavior of these terms clearly suggests that their energy dependence can obviously be reproduced by other simpler functions within the finite incerval of practical interest. It is well known in numerical analysis that the rational functions are best suited to approximate a well behaved function within a finite range. Hence, the obvious choice is to set the approximate functions $\hat{S}_{i}^{(x)}(\sqrt{E})$ and $\hat{q}_{l}^{(x)}(\sqrt{E})$ co be rational functions of arbitrary order. If the order of polynomials in the numeracor and the denominator are taken to be 34 and $N$ IN Iespec. tively, a total of ( $M M+N N+2)$ complex coefficients (twice as many entries) can be used as the variables to be determined in the non-linear fitting procedure. Because of the extremely well behaved nature of $s_{l}^{(x)}(\sqrt{E})$ and $q_{l}^{(x)}(\sqrt{E})$, the MH and $N$ required are expected to be very small. One atcractive feature of the proposed method is that the rational functions so obtained can be again expressed in the form of pole expansion via partial fraction, i.e.,

$$
\begin{aligned}
& \hat{s}_{i}^{(x)}(\sqrt{E})=\frac{P_{2 M 1}(\sqrt{E})}{Q_{2 a 1}(\sqrt{E})}=\sum_{i=1}^{N N} \frac{I_{\lambda}^{(x)}(-i)}{\alpha_{i}-\sqrt{E}} \\
& \hat{q}_{i}^{(x)}(\sqrt{E})=\sum_{i=1}^{N N} \frac{b_{i}^{(x)}(-i)}{\xi_{i}-\sqrt{E}}
\end{aligned}
$$

if $\mathrm{NN}>\mathrm{MM}$

Conceptually, the procedure is equivalent to determining the analytic continuations of $S_{l}^{(x)}(\sqrt{E})$ and $q_{l}^{(x)}(\sqrt{E})$ for $\sqrt{E_{1}} \leq \sqrt{E} \leq \sqrt{E_{u}}$. The pole and residue parameters so obtained can be viewed as 'pseudo' pole parameters. A code (htOPJR) based on the MINPACK-package $e^{s}$ has been developed to compute these 'pseudo' pole parameters. To provide sufficient accuracy to cross sections, ki of no greater than 3 is required.

The Doppler-broadening of Eq. 3 and Eq. 5 immediately leads to the similar form defined by the traditional formalisms:

$$
\begin{aligned}
& \sigma_{x}(E, T)=\frac{1}{E} \sum_{t} \mathscr{R e}\left\{\sum_{\xi} \sum_{i=1}^{N} 2 \sqrt{E} R_{l, J, 1, \lambda}^{(x)} \frac{\sqrt{\pi}}{\Delta_{z}} w\left(\frac{E-\varepsilon_{\dot{i}}}{\Delta_{z}}\right)\right. \\
& \left.+s_{\imath}^{(x)}(\sqrt{E}, T)+\hat{q}_{i}^{(x)}(E) \cdot \delta_{l}\right\}
\end{aligned}
$$

where

$$
\begin{aligned}
& S_{l}^{(x)}(\sqrt{E}, T)=\sum_{x=1}^{m \pi} \frac{I_{i k}^{(x)}}{\Delta_{m}}\left[\sqrt{\pi} W\left(\frac{\sqrt{E}-\alpha_{k}^{*}}{\Delta_{m}}\right)\right] \\
& \epsilon_{\lambda}=\left[p_{\lambda}^{(1)+]^{2}}\right. \\
& \Psi(x, y)+i \chi(x, y)=\sqrt{\pi} \text { y W }(z)
\end{aligned}
$$

and $\Delta_{z}=2 \sqrt{E} \Delta_{\eta \pi}$ denotes the Doppler width in energy and momentum domains respectively.

Thus, the only difference between Eq. 7 and the traditional formalisms is the presence of the 'background' terms explicitly defined by a handful of 'pseudo' poles with weak energy and temperacure dependence. Its compatibility to all ENDF/B format based codes is quite apparent.

\section{Results and Conclusions}

Extensive calculations have been carriad out in order to demonstrate the viability or the proposed method. The studies include ali major actinides and two structural isocopes.

The viability of the method can be best illustrated by exanining the relative errors in the resulting cross sections with respect to tine 
directly computed Reich-Moora cross sections. Representative results corresponding to the absorption crass sections of ${ }^{239} \mathrm{Pu}$ and ${ }^{238} \mathrm{U}$ are show in Fig. 1 and Fig. 2 respectively. These figures show the behavior of the absolute values of relative eriors in great detail as a function of $\sqrt{E}$ over the $\left\{\sqrt{E_{f}} \leq \sqrt{E} \leq \sqrt{E_{u}}\right)$. By and large,

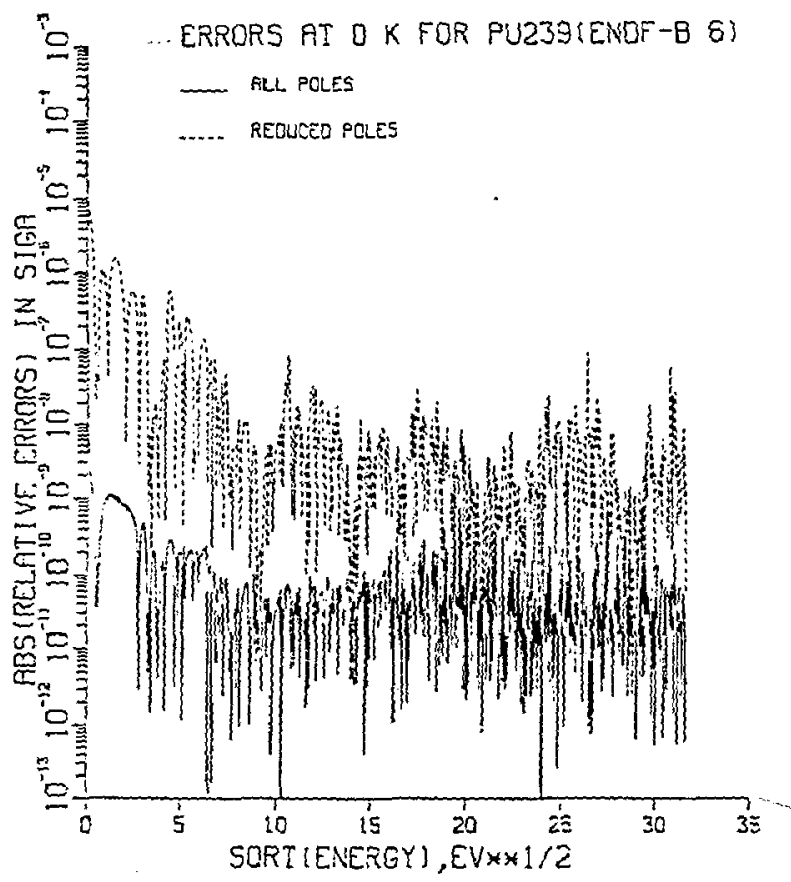

Fig. 1. Relacive errors in o, of $239 \mathrm{u}$ based on the parameters converted from ENDF/B VI dara.

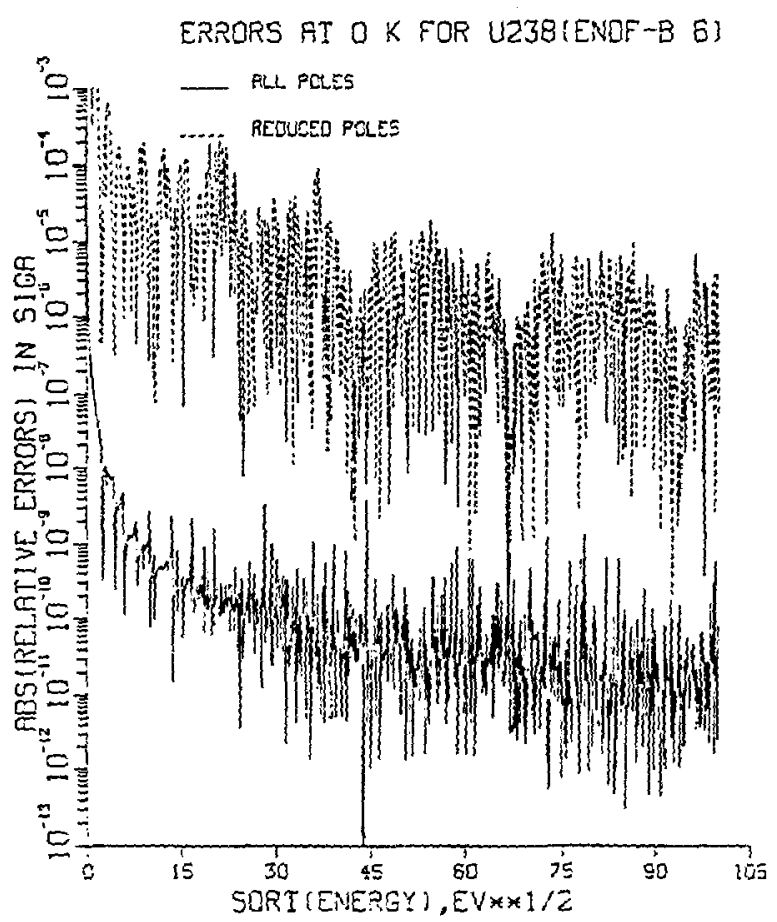

Fig. 2 the relative errors and the cross section values are anti-correlated. The peaks of the former correspond to the valleys of the latter and vice versa. The lower curves represent che inevitable errors attributed to the compucations of parameters when all poles defined by Eq. 1 are included. The errors are generally less than $10^{-9}$ and can reach $10^{-7}$ as $\mathrm{E}$ approaches zeco. Thus, Eq. 1 is not only analytically rigorous but also numerically exact for practical purposes. The upper curves show the corresponding errors when the simplified version is used. It is imporcant to note that the results ate based on the tolerance limit of $10^{-5}$ and $N N-3$ in computing the background terms. The maximum errors are of the order of $10^{-4}$ near the valleys between resonances (much smaller for fissionable nuclides) and are clearly coler. able in practical applications.

If is, therefore, reasonable to conclude that the proposed method is not only readily amenable to methodologies based on the czacizional formalisms but also preserves the rigor of the Relch-Hoore cross sections. The procedure is being implewented in the $\mathrm{MC}^{2}-2$ code. ${ }^{6}$

\section{References}

1. P. F. Rose and C. L. Dunford, Ed., 'ENDF-102 Data Formats and Procedures for Evaluated Nuclear Data File ENDF-6,' Biz-\$C5-44945 (1990).

2. C. H. Reich and M. S. Hoore, Phys. Rev. I11, 929 (1958).

3. R. N. Hwang, Nucl, ScL, Engr, 95, 192 (1987).

4. J. Humblat and L. Rosenfeld, Nucl. Phys, 26, 529 (1961).

5. J. J. Moré, B. S. Garbow and K. E. H. Millstrom, 'User Guide for MTNPACK-1,' ANL. $80-74(1980)$.

6. H. Henryson, B. J. Toppel and C. G. Stenberg, 'Mc'2: A Code to Calculace Fast Neutron Spectra and Multígroup Cross Sections," ANL+8144 (1976). 